\title{
A SPECTRAL THEORY FOR INNER FUNCTIONS
}

BY

\section{MALCOLM J. SHERMAN}

Let $\mathscr{U}$ be an inner function in the sense of Lax; i.e., $\mathscr{U}\left(e^{i \theta}\right)$ is almost everywhere a unitary operator on a separable Hilbert space $\mathscr{H}$, and $\mathscr{U}$ belongs weakly to the Hardy class $H^{2}$. Inner functions arise from subspaces of $H_{\mathscr{H}}^{2}$ invariant under the right shift operator ("invariant subspaces") and from bounded operators on $\mathscr{H}$ in ways to be specified. We are interested in finding canonical forms for and invariants of inner functions, and where an inner function comes from an operator on $\mathscr{H}$, in relating the invariants of the inner function to those of the operator. For basic definitions and notations consult [5, particularly Chapter VI], or [15].

1. Eigenfunctions. We will say that an inner function $\mathscr{U}$ is finite dimensional if the underlying Hilbert space $\mathscr{H}$ is finite dimensional. The simplest inner functions are then finite dimensional diagonal ones; i.e., of the form

$$
\mathscr{U}=\left(\begin{array}{llll}
q_{1} & & & 0 \\
& q_{2} & & \\
& & \ddots & \\
0 & & & q_{n}
\end{array}\right)
$$

where the $q$ 's are inner functions in the sense of Beurling, which we will call scalar inner functions. The $q$ 's look like eigenvalues and we begin with the following definition.

DEFINITION 1.1. A scalar inner function $q$ is an eigenfunction of an inner function $\mathscr{U}$ if the set of $z$ in the disk $|z| \leqq 1$ such that $\mathscr{U}(z)-q(z) I$ has a bounded inverse is at most a set of linear measure 0 on the circle $\{z:|z|=1\}$. The set of such $q$ 's will be called the spectrum of $\mathscr{U}$.

If $\mathscr{U}$ is finite dimensional this condition reduces to det $(\mathscr{U}-q I) \equiv 0$ (where the boundary value function need vanish only almost everywhere). Since an $H^{2}$ function which vanishes on a set of positive measure on the circle vanishes identically it would suffice to assume $\mathscr{U}-q I$ not invertible on a set of positive measure on the circle. If $\mathscr{H}$ is infinite dimensional the two conditions are not equivalent as will be shown later. (See the comments following Corollary 3.6.) Notice that $q$ is determined uniquely as a point function for $|z|<1$, and not just up to a scalar factor of modulus 1 .

The first point to be made about eigenfunctions is that one should not expect them always to exist. For example, if $\mathscr{H}$ is 2 dimensional any eigenfunction $q$ of

Presented to the Society, in part, January 24, 1968 under the title The eigenfunctions of certain inner functions; received by the editors August 25, 1967. 
$\mathscr{U}$ is a solution of $q^{2}-(\operatorname{tr} \mathscr{U}) q+\operatorname{det} \mathscr{U}=0$. For each $z, q(z)$ is given explicitly by the formula for the solution of a quadratic, and $q$ will be analytic for $|z|<1$ if and only if all roots in the interior of the disk of the discriminant $(\operatorname{tr} \mathscr{U})^{2}-4 \operatorname{det} \mathscr{U}$ are of even order. One can write down inner functions where this fails. For example, it is not the case for appropriate choices of $\lambda$ and $\mu$ if we take

$$
\mathscr{U}=\left(\begin{array}{cc}
\frac{q_{\lambda}}{\sqrt{ } 2} & \frac{q_{\lambda} q_{\mu}}{\sqrt{ } 2} \\
-\frac{1}{\sqrt{ } 2} & \frac{q_{\mu}}{\sqrt{ } 2}
\end{array}\right)
$$

where $q_{\lambda}(z)$ is the Blaschke factor $(z-\lambda)(1-\lambda z)^{-1}$. From a more general point of view we are looking for roots of polynomials over the ring of $H^{\infty}$ functions, and the latter are far from being algebraically closed. However, just as selfadjoint real matrices have eigenvalues we might expect important classes of inner functions to possess eigenfunctions.

The most natural questions then are which inner functions are similar to diagonal inner functions and which have eigenfunctions. We answer the first question completely (Theorem 2.5) after giving an infinite dimensional generalization (Definition 2.1) of the concept of a diagonal inner function. We answer the second question. when $\mathscr{H}$ is finite dimensional (Theorem 5.6) and are able to characterize those finite dimensional inner functions the sum of the multiplicities of whose eigenfunctions is maximal (Theorem 5.4). We also determine the spectrum of certain inner functions which arise naturally from bounded operators on $\mathscr{H}$ (Theorems 3.4 and 3.5), and give a simpler condition than Theorem 2.5 for these to be of scalar type (Theorem 4.3).

\section{Inner functions of scalar type.}

Definition 2.1. An inner function $\mathscr{U}$ is said to be of scalar type if there exist a Borel function $f(z, \phi)$ and a spectral measure $E$ on the unit circle such that

$$
\mathscr{U}(z)=\int_{0}^{2 \pi} f(z, \phi) d E(\phi), \quad|z| \leqq 1 .
$$

For the above integral to be defined it is sufficient that $f(z, \phi)$ be measurable in $\phi$ for fixed $z$. However, in order to conclude that $f$ has some analytic structure we need the Fubini theorem and so assume joint measurability.

Proposition 2.2. Let $E$ be a spectral measure on the circle and let $f(z, \phi)$ be a Borel function such that the functions $f_{\phi}(z)=f(z, \phi)$ are scalar inner functions except possibly for $\phi$ in a set of $E$ measure 0 . Then $\mathscr{U}(z)=\int f(z, \phi) d E(\phi),|z| \leqq 1$, is an inner function. Conversely, if $f(z, \phi)$ is Borel and $\mathscr{U}(z)=\int f(z, \phi) d E(\phi)$ is inner, there exists a Borel function $g(z, \phi)$ such that

(i) $f\left(e^{i \theta}, \phi\right)=g\left(e^{i \theta}, \phi\right)$,

(ii) for fixed $z, g(z, \phi)=f(z, \phi)$ except possibly for $\phi$ in a set of $E$ measure 0; i.e., $g$ induces the same $\mathscr{U}$, 
(iii) the functions $g_{\phi}(z)=g(z, \phi)$ are scalar inner functions except possibly for $\phi$ in a set of $E$ measure 0.

Proof. The Fubini theorem implies that $\int \mathscr{U}\left(e^{i \theta}\right) e^{n i \theta} d \theta=0$ for $n>0$. Since $f\left(z_{n}, \phi\right)$ converges boundedly to $f(z, \phi)$ as $z_{n}$ converges to $z$ we see that $\mathscr{U}(z)$ is continuous when $|z|<1$. Morera's theorem and another application of the Fubini theorem imply that $\mathscr{U}(z)$ is analytic for $|z|<1$. Since $f\left(r e^{i \theta}, \phi\right)$ converges a.e. to $f\left(e^{i \theta}, \phi\right)$ for almost all $\phi[E], \mathscr{U}(z)$ must be the analytic extension to the disk of $\mathscr{U}\left(e^{i \theta}\right)$. Conversely if $\mathscr{U}(z)=\int f(z, \phi) d E(\phi)$ is inner the Fubini theorem implies that $\int f\left(e^{i \theta}, \phi\right) e^{n i \theta} d \theta=0$ for $n>0$ for almost all $\phi[E]$. Since $\mathscr{U}^{*}(z)=\int \bar{f}\left(e^{i \theta}, \phi\right) d E(\phi)$ we must have $\left|f\left(e^{i \theta}, \phi\right)\right|=1$ a.e. in $\theta$ for almost all $\phi[E]$. We then, following a suggestion of the referee's, define $g(z, \phi)$ to be the analytic extension to the disk of $f\left(e^{i \theta}, \phi\right)$. Since

$$
g(z, \phi)=\frac{1}{2 \pi i} \int_{|\zeta|=1} \frac{f(\zeta, \phi)}{\zeta-z} d \zeta, \quad|z|<1
$$

$g$ is jointly measurable and by the above induces an inner function with the same boundary values as $\mathscr{U}$.

If $\mathscr{U}$ is of scalar type clearly $\mathscr{U}(z) \mathscr{U}(w)=\mathscr{U}(w) \mathscr{U}(z)$ for $|z|,|w| \leqq 1$. We now prove the converse.

LEMMA 2.3. Let $\mathscr{U}$ be an inner function such that $\mathscr{U}\left(e^{i \theta}\right) \mathscr{U}\left(e^{i \phi}\right)=\mathscr{U}\left(e^{i \phi}\right) \mathscr{U}\left(e^{i \theta}\right)$ for almost all $\theta, \phi$. Then the Fourier coefficients of $\mathscr{U}$ are a commuting family of normal operators.

Proof. Let $\mathscr{U}(z)=\sum_{n=0}^{\infty} T_{n} z^{n}$. Then $T_{n}=(1 / 2 \pi) \int_{0}^{2 \pi} \mathscr{U}\left(e^{i \theta}\right) e^{-n i \theta} d \theta$, where we can take the integrals in the weak sense. One easily checks that for all $x, y \in \mathscr{H}$

$$
\left(T_{n} T_{m} x, y\right)=\frac{1}{4 \pi^{2}} \iint\left(\mathscr{U}\left(e^{i \phi}\right) \mathscr{U}\left(e^{i \theta}\right) x, y\right) e^{-n i \phi} e^{-m i \theta} d \phi d \theta
$$

which implies $T_{n} T_{m}=T_{m} T_{n}$. We also have

$$
\begin{aligned}
& \left(T_{n} T_{n}^{*} x, y\right)=\frac{1}{4 \pi^{2}} \iint\left(\mathscr{U}\left(e^{i \phi}\right) \mathscr{U}\left(e^{i \theta}\right)^{*} x, y\right) e^{n i(\theta-\phi)} d \phi d \theta \\
& \left(T_{n}^{*} T_{n} x, y\right)=\frac{1}{4 \pi^{2}} \iint\left(\mathscr{U}\left(e^{i \theta}\right)^{*} \mathscr{U}\left(e^{i \phi}\right) x, y\right) e^{n i(\theta-\phi)} d \phi d \theta .
\end{aligned}
$$

These are equal since

$$
\mathscr{U}\left(e^{i \theta}\right)^{*} \mathscr{U}\left(e^{i \phi}\right) \mathscr{U}\left(e^{i \theta}\right) \mathscr{U}\left(e^{i \theta}\right)^{*}=\mathscr{U}\left(e^{i \theta}\right)^{*} \mathscr{U}\left(e^{i \theta}\right) \mathscr{U}\left(e^{i \phi}\right) \mathscr{U}\left(e^{i \theta}\right)^{*} .
$$

If one assumes the equivalence in a separable Hilbert space of the weak integral and various more direct approaches to integration, a perhaps more illuminating proof of the lemma results from the observations:

(i) a finite linear combination of commuting normal operators is normal; 
(ii) the integral for $T_{n}$ is the limit in norm of finite linear combinations of the $\mathscr{U}\left(e^{i \theta}\right)$ 's.

COROLlaRY 2.4. If $\mathscr{U}$ satisfies the hypothesis of the lemma then

(i) $\mathscr{U}(z)$ is normal for $|z|<1$,

(ii) $\mathscr{U}(z) \mathscr{U}(w)=\mathscr{U}(w) \mathscr{U}(z)$ for $|z|,|w|<1$.

THEOREM 2.5. An inner function $\mathscr{U}$ is of scalar type if and only if $\mathscr{U}\left(e^{i \theta}\right) \mathscr{U}\left(e^{i \phi}\right)$ $=\mathscr{U}\left(e^{i \phi}\right) \mathscr{U}\left(e^{i \theta}\right)$ for almost all $\theta, \phi$.

Proof. Let $\mathscr{U}(z)=\sum_{n=0}^{\infty} T_{n} z^{n}$. By the lemma the $T_{n}$ 's are normal and commute. By a well-known theorem $[14$, p. 36$]$ of von Neumann there exists a self adjoint operator $T$ such that every $T_{n}$ is a bounded Borel function of $T$. Let $U=(T-i I)$ $\cdot(T+i I)^{-1}$, the Cayley transform of $T . U$ is unitary, $1 \notin \sigma(U)[2$, p. 569] and $T=i(I+U)(I-U)^{-1}$. Thus $T=g(U)$, where $g(\lambda)=i(1+\lambda)(1+\lambda)^{-1}$ and every $T_{n}$ is a bounded (on $\sigma(U)$ ) Borel function $f_{n}$ of $U$. Thus

$$
\mathscr{U}(z)=\sum_{n=0}^{\infty} f_{n}(U) z^{n}=\sum_{n=0}^{\infty} \int f_{n}(\phi) z^{n} d E(\phi) .
$$

Since $\left\|T_{n}\right\|=E$ - ess sup $\left|f_{n}\right|=\left\|(1 / 2 \pi) \int \mathscr{U}\left(e^{i \theta}\right) e^{-n i \theta} d \theta\right\| \leqq 1$, we can invert the order of integration and summation for $|z|<1$, getting

$$
\mathscr{U}(z)=\int f(z, \phi) d E(\phi), \quad|z|<1
$$

where $f(z, \phi)=\sum f_{n}(\phi) z^{n}$. Since

$$
\|\mathscr{U}(z)\|=E \underset{\phi}{\operatorname{ess} \sup }|f(z, \phi)|<1
$$

$f_{\phi}(z)$ is a bounded analytic function for almost all $\phi[E]$. We can thus extend $f_{\phi}(z)$ to $f_{\phi}\left(e^{i \theta}\right)$. For all $x, y \in \mathscr{H}$

$$
\begin{aligned}
\left(\mathscr{U}\left(e^{i \theta}\right) x, y\right) & =\lim _{r \rightarrow 1}\left(\mathscr{U}\left(r e^{i \theta}\right) x, y\right)=\lim _{r \rightarrow 1} \int f\left(r e^{i \theta}, \phi\right) d(E(\phi) x, y) \\
& =\int f\left(e^{i \theta}, \phi\right) d(E(\phi) x, y),
\end{aligned}
$$

and therefore $\mathscr{U}(z)=\int f(z, \phi) d E(\phi)$ for $|z| \leqq 1$.

3. Operators and inner functions. We next show that there is a reasonably large supply of inner functions of scalar type. If $T$ is a bounded operator on the underlying Hilbert space, $\|T\|<1$, there are at least two natural ways of associating to $T$ an inner function. One, due to Rota [13] and Lowdenslager, assigns to $T$ any inner function $\mathscr{U}_{T}$ corresponding to the invariant subspace of $H_{\mathscr{H}}^{2}$ which is the orthogonal complement in $H_{\mathscr{H}}^{2}$ of all elements of the form

$$
e+(T e) z+\left(T^{2} e\right) z^{2}+\left(T^{3} e\right) z^{3}+\cdots
$$


where $e \in \mathscr{H}$ (the underlying Hilbert space). The other, due to Potapov [12, p. 145], involves the explicit formula

$$
\mathscr{V}_{T}(z)=\left(I-T^{*} T\right)^{-1 / 2}\left(z-T^{*}\right)(I-z T)^{-1}\left(I-T T^{*}\right)^{1 / 2} .
$$

It is known [15] that if $T$ is normal, a Rota inner function is given by $\mathscr{U}_{T}(z)$ $=\left(z-T^{*}\right)(I-z T)^{-1}$ and, of course, $\mathscr{V}_{T}$ reduces to this when $T$ is normal.

Corollary 3.1. $\mathscr{V}_{T}$ is of scalar type if $T$ is normal.

Proof. Let $T$ have spectral measure $P$. Standard properties of spectral integrals $[4$, p. 61] imply

$$
\mathscr{V}_{T}(z)=\int_{|\lambda| \leqq 1} \frac{z-\bar{\lambda}}{1-\lambda z} d P(\lambda)
$$

and that the values of $\mathscr{V}_{T}$ commute.

If $T$ is selfadjoint it is easy to visualize the representation of $\mathscr{V}_{T}$ as $\int f(z, \phi) d E(\phi)$. One simply transfers the spectral measure $P$ of $T$ from the real axis to the circle (or uses the spectral measure of the Cayley transform) and then

$$
\mathscr{V}_{T}(z)=\int_{0}^{2 \pi} \frac{z-\phi}{1-\phi z} d P(\phi)
$$

for $P$ thus modified. If $T$ is normal but not selfadjoint one needs the full strength of the theorem. For example, let $T$ have spectrum $\sigma(T)=\left\{\lambda:|\lambda| \leqq \frac{1}{2}\right\}$. Then $\sigma\left(\mathscr{V}_{T}(z)\right)$ is the image of $\sigma(T)$ under the map $\lambda \rightarrow(z-\bar{\lambda})(1-\lambda z)^{-1}$. But

$$
\sigma\left(\mathscr{V}_{T}(z)\right)=\sigma\left(f_{z}(U)\right)=f_{z}(\sigma(U)) \text {, }
$$

where by $f_{z}(\sigma(U))$ we mean the essential range [4, p. 52] of $f_{z}$ on $\sigma(U)$, the notations ( $f$ and $U$ ) coming from the proof of Theorem 2.5. Thus for fixed $z$ the function $f(z, \phi)$ maps the unit circle $\{z:|z|=1\}$ onto a set with interior. An explicit construction would present difficulties.

For an inner function $\mathscr{U}, \sigma(\mathscr{U})$ will denote the set of eigenfunctions (Definition 1.1) of $\mathscr{U}$. If $T$ is an operator $\sigma(T)$ is the spectrum of $T$. If one identifies a constant function with its range these definitions are consistent for $T$ a unitary operator (constant inner function).

THEOREM 3.2. If $T$ is normal, $\|T\|<1$,

$$
\sigma\left(\mathscr{V}_{T}\right) \supset\left\{q_{\lambda}: \lambda \in \sigma(T)\right\}
$$

where $q_{\lambda}(z)$ is the Blaschke factor $(z-\lambda)\left(1-\lambda_{z}\right)^{-1}$.

Proof.

$$
\mathscr{V}_{T}(z)=\int(z-\lambda)(1-\lambda z)^{-1} d P(\lambda),
$$

where $P$ is the spectral measure of $T$. If $\mu \in \sigma(T)$ then for fixed $z$

$$
g(\lambda)=\frac{z-\bar{\lambda}}{1-\lambda z}-\frac{z-\bar{\mu}}{1-\mu z}
$$


is a continuous function of $\lambda$ which vanishes at a point of $\sigma(T)$ and therefore

is not invertible.

$$
\mathscr{V}_{T}(z)-q_{\bar{\mu}}(z) I=\int_{|\lambda| \leqq 1}\left[\frac{z-\bar{\lambda}}{1-\lambda z}-\frac{z-\bar{\mu}}{1-\mu z}\right] d P(\lambda),
$$

THEOREM 3.3. If $T$ is selfadjoint, $\|T\|<1$, then $\sigma\left(\mathscr{V}_{T}\right)=\left\{q_{\lambda}: \lambda \in \sigma(T)\right\}$.

Proof. If $q \in \sigma\left(\mathscr{V}_{T}\right)$ then for every $z$ there is a (real) number $\lambda(z) \in \sigma(T)$ such that

$$
\frac{z-\lambda(z)}{1-z \lambda(z)}-q(z)=0
$$

Solving for $\lambda$ we get $\lambda(z)=[z-q(z)][1-z q(z)]^{-1}$. This is a real analytic function and therefore constantly equal to $\mu$, where $\mu \in \sigma(T)$. Clearly then

$$
q(z)=(z-\mu)(1-\mu z)^{-1} .
$$

THEOREM 3.4. If $T$ is normal and $q \in \sigma\left(\mathscr{V}_{T}\right)$ then there exists $\mu \in \sigma(T)$ and a complex number $\alpha,|\alpha|=1$, such that

$$
q(z)=\alpha \frac{z-\bar{\mu}}{1-\mu z} .
$$

Proof. As above, if $q \in \sigma\left(\mathscr{V}_{T}\right)$ there is a function $\lambda(z)$ whose range is contained in $\sigma(T)$ such that

Let $\|T\|=1-\varepsilon$. Then

$$
\frac{z-\lambda(z)}{1-z \lambda(z)}=q(z)
$$

$$
|q(z)| \geqq \frac{|z|-(1-\varepsilon)}{1+|z|(1-\varepsilon)}
$$

and if $|z| \geqq 1-\varepsilon / 2$ we have $|q(z)| \geqq \varepsilon / 4$. Thus $q$ has no zeros with modulus $\geqq 1-\varepsilon / 2$ and therefore only finitely many zeros. Using the known structure [8, p. 67] of inner functions it is easy to see that if $q$ has a singular part corresponding to a measure $\nu$, and $e^{i \theta}$ is in the support of $\nu$, then $\liminf _{r \rightarrow 1} q\left(r e^{i \theta}\right)=0$. Thus $q$ is a finite Blaschke product. Clearly $q(\mu)=0$ if and only if $\bar{\mu}=\lambda(\mu)$ and thus the zeros of $q$ are in $\sigma\left(T^{*}\right)$. It remains to show that $q$ has only one zero. Solving simultaneously the equations

$$
\begin{aligned}
\lambda(z)-z q(z) \lambda(z) & =z-q(z) \\
-\bar{z} \bar{q}(z) \bar{\lambda}(z)+\lambda(z) & =\bar{z}-\bar{q}(z),
\end{aligned}
$$

we get

$$
\lambda(z)=\frac{\bar{z}\left[1-|q(z)|^{2}\right]-\bar{q}(z)\left[1-|z|^{2}\right]}{1-|z q(z)|^{2}} .
$$

Thus for $|z|<1, \lambda(z)$ is uniquely determined by $q(z)$ and depends continuously on $z$. Clearly

$$
|\lambda(z)| \geqq \frac{|z-q(z)|}{1+|z q(z)|} \geqq \frac{|z-q(z)|}{2}, \quad|z|<1 .
$$


If $\sup _{|z| \leqq 1}|z-q(z)|=2$, then $q \notin \sigma\left(\mathscr{V}_{T}\right)$ since $\lambda(z) \in \sigma(T) \subset\{z:|z| \leqq\|T\|=1-\varepsilon\}$. Obviously $\sup _{|z| \leqq 1}|z-q(z)|=2$ is equivalent to the existence of $\theta$ such that $q\left(e^{i \theta}\right)=-e^{i \theta}$, which is to say that $-q$ has a fixed point on $|z|=1$. It follows from the Lefschetz fixed point theorem [11, p. 157] that if $q$ has degree $\neq 1$ (i.e., if $q(z)$ has more than one zero inside the disk) then $q$ has a fixed point on the circle. An analytic proof can be given as follows. Define

$$
g(\theta)=\arg q\left(e^{i \theta}\right)-(\theta+2 \pi) .
$$

Then $g(0)<0$ and $g(2 \pi) \geqq 0$ if $q$ has more than one zero. Clearly $g(\theta)=0$ is equivalent to $q\left(e^{i \theta}\right)=e^{i \theta}$.

The geometrical nature of the fact that sup $|z-q(z)|=2$ emerged during a conversation with $\mathrm{Mr}$. Michael Behrens, for which observation the author would like to express his thanks to Mr. Behrens.

We observe that one can have $\alpha q_{\lambda} \in \sigma\left(\mathscr{V}_{T}\right)$ for $\alpha \neq 1$. For example, if $T$ has spectrum $\{\lambda:|\lambda| \leqq 1 / 2\}$ and we put $q(z)=\alpha z$, then by $\left(^{*}\right)$

$$
|\lambda(z)|=\frac{|(1-\bar{\alpha}) \bar{z}|}{1+|z|^{2}} \leqq \frac{1}{2}|1-\alpha| .
$$

Thüs if $|1-\alpha| \leqq 1, q \in \sigma\left(\mathscr{V}_{T}\right)$. This phenomenon depends only on the geometry of $\sigma(T)$, and mostly on the distance from a point $\mu \in \sigma\left(T^{*}\right)$ to $\rho\left(T^{*}\right)$.

THEOREM 3.5. Let $T$ be normal with $\bar{\mu} \in \sigma(T)$ and let $\gamma=\inf _{z \notin \sigma\left(T^{*}\right)}|\mu-z|$. Then

(i) $\alpha q_{\mu} \in \sigma\left(\mathscr{V}_{T}\right)$ if $|1-\alpha| \leqq \gamma$,

(ii) $\alpha q_{\mu} \notin \sigma\left(\mathscr{V}_{T}\right)$ if $|1-\alpha||\mu|>\gamma$ and $|1-\alpha||1-\mu|>2 \gamma$.

Proof. Let $q=q_{\mu}$ and let $\lambda$ be the function $\left(^{*}\right)$ corresponding to $\alpha q$. Then by $\left(^{*}\right)$

$$
\begin{aligned}
\lambda(z)-\mu & =\frac{\bar{z}\left[1-|q(z)|^{2}\right]-\bar{\alpha} \bar{q}(z)\left[1-|z|^{2}\right]}{1-|z q(z)|^{2}}-\frac{\bar{z}\left[1-|q(z)|^{2}\right]-\bar{q}(z)\left[1-|z|^{2}\right]}{1-|z q(z)|^{2}} \\
& =(1-\bar{\alpha}) \bar{q}(z) \frac{1-|z|^{2}}{1-|z q(z)|^{2}} .
\end{aligned}
$$

Thus $|\lambda(z)-\mu| \leqq|1-\alpha|$, which proves (i). Since

$$
\inf _{|z|=r}|q(z)|=\left|\frac{r-|\mu|}{1-|\mu| r \mid}\right|
$$

we get

$$
\left|\frac{\bar{q}(z)\left[1-|z|^{2}\right]}{1-|z q(z)|^{2}}\right| \geqq \frac{(r-|\mu|)(1-|\mu| r)}{1-2|\mu| r+r^{2}}=g(r) .
$$

By elementary calculus $g$ has no relative extreme points for $0<r<1$. We note that $|g(0)|=|\mu|$ and $g(1)=(1-|\mu|) / 2$ from which (ii) follows.

COROLlaRY 3.6. If $T$ is normal and $\sigma(T)$ has no interior then

$$
\sigma\left(\mathscr{V}_{T}\right)=\left\{q_{\lambda}: \lambda \in \sigma\left(T^{*}\right)\right\} .
$$


In particular, the conclusion holds if $T$ is selfadjoint (Theorem 3.3 ) or if $\mathscr{H}$ is finite dimensional.

A few general remarks seem appropriate here. Had we defined $\sigma(\mathscr{U})$ by the requirement that $\mathscr{U}\left(e^{i \theta}\right)-q\left(e^{i \theta}\right) I$ not be invertible on a set of positive measure on the circle we would get the same finite dimensional theory, although Theorems 3.3 to 3.6 would fail using this definition, as we now show. If $T$ has real spectrum then (see Theorem 3.3)

$$
\lambda(z)=\frac{z-q(z)}{1-z q(z)}
$$

If we take $q(z)=z^{2}$ then $\lambda\left(e^{i \theta}\right)$ is real and it is easy to see that $\left|\lambda\left(e^{i \theta}\right)\right| \leqq 1-\varepsilon$ on a set of positive measure, and also $\left|\lambda\left(e^{i \theta}\right)\right| \geqq 1-\varepsilon$ on a set of positive measure. Thus for appropriate selfadjoint $T$ we would have $z^{2} \in \sigma\left(\mathscr{V}_{T}\right)$ if we used the weakened definition. Another possible definition of $\sigma(\mathscr{U})$, and a natural one considering that $\mathscr{U}(z)$ is completely determined by $\mathscr{U}\left(e^{i \theta}\right)$, is to require that $\mathscr{U}\left(e^{i \theta}\right)-q\left(e^{i \theta}\right) I$ be invertible almost nowhere on the circle, with nothing said about the situation inside the disk. We do not know if this definition is equivalent. If $T$ is selfadjoint, then $\sigma\left(\mathscr{V}_{T}\right)$ $=\left\{q_{\lambda}: \lambda \in \sigma(T)\right\}$ using this last definition, which we now prove. We observe that $1-z q(z)$ is bounded and (as observed to the author by Helson) has positive real part and is therefore outer $\left[8\right.$, p. 76]. Thus $\lambda(z) \in H^{2}$, and since it has real boundary values it must be constant. Thus Theorem 3.3 would remain true using the weakened definition. Whether or not Theorem 3.4 would remain true is open.

Another definition of the spectrum of an inner function $\mathscr{U}$ is the set of all complex numbers $\lambda$ such that

(i) $\mathscr{U}(\bar{\lambda})$ is not invertible if $|\lambda|<1$, or

(ii) $\mathscr{U}(z)$ cannot be continued analytically past $\lambda$ if $|\lambda|=1$.

This set is known to be the spectrum of the restriction to $\mathscr{K}$ of the adjoint $S^{*}$ of the right shift operator $S$ acting in $H_{\mathscr{H}}^{2}$, where $\mathscr{K}$ is the complement in $H_{\mathscr{H}}^{2}$ of $\mathscr{U} H_{\mathscr{H}}^{2}$. (This characterization is due to Lax and Phillips [10] for $|\lambda|<1$ and to Helson, Srinivasan and Wang [5, p. 74], in the general case.) It is clear that if $\mathscr{U}$ has a scalar inner function $q$ in its spectrum and $q\left(\lambda_{1}\right)=q\left(\lambda_{2}\right)=\cdots=0$, then $\bar{\lambda}_{1}, \bar{\lambda}_{2}, \ldots$ are in the spectrum of $\mathscr{U}$ in Helson's sense. To illustrate the difference: if $\mathscr{H}$ is 2 dimensional and we let

$$
\mathscr{U}(z)=z\left(\frac{z-\frac{1}{2}}{1-\frac{1}{2} z}\right) I \quad \text { and } \quad \mathscr{U}(z)=\left(\begin{array}{cc}
z & 0 \\
0 & \frac{z-\frac{1}{2}}{1-\frac{1}{2} z}
\end{array}\right)
$$

then the scalar spectrum of both $\mathscr{U}$ and $\mathscr{V}$ is $\left\{0, \frac{1}{2}\right\}$, whereas in our sense $\mathscr{U}$ has spectrum $q(z)=z\left(z-\frac{1}{2}\right)\left(1-\frac{1}{2} z\right)^{-1}$ and $\mathscr{V}$ has spectrum $q_{1}(z)=z$ and $q_{2}(z)=$ $\left(z-\frac{1}{2}\right)\left(1-\frac{1}{2} z\right)^{-1}$. Thus the spectrum in our sense can be both more delicate, as in the example, and less delicate, as when it is empty.

4. Adjoints of inner functions. All examples of well behaved inner functions have so far come from normal operators. The latter are defined by the property of 
commuting with an adjoint. It is natural then to seek an adjoint for inner functions. Lemma 4.2 tends to justify the definition and Theorem 4.3 gives a simpler condition than that of Theorem 2.5 for Potapov inner functions to be of scalar type.

Definition 4.1. Let $\tilde{\mathscr{U}}(z)=\mathscr{U}(\bar{z})^{*}$.

It is easy to check that $\int \tilde{\mathscr{U}}\left(e^{i \theta}\right) e^{n i \theta} d \theta=0, n=1,2, \ldots$, if $\mathscr{U}$ is inner.

Lemma 4.2. $\tilde{\mathscr{V}}_{T}=\mathscr{V}_{T^{*}}$, where $\mathscr{V}_{T}$ is the Potapov inner function

$$
\left(I-T^{*} T\right)^{-1 / 2}\left(e^{i \theta}-T^{*}\right)\left(I-e^{i \theta} T\right)^{-1}\left(I-T T^{*}\right)^{1 / 2} .
$$

Proof.

$$
\begin{aligned}
& \mathscr{V}_{T^{*}}-\tilde{\mathscr{V}}_{T}=\left(I-T T^{*}\right)^{-1 / 2}\left[\left(e^{i \theta}-T\right)\left(I-e^{i \theta} T^{*}\right)^{-1}\left(I-T^{*} T\right)\right. \\
&\left.-\left(I-T T^{*}\right)\left(I-e^{i \theta} T^{*}\right)^{-1}\left(e^{i \theta}-T\right)\right]\left(I-T^{*} T\right)^{-1 / 2} .
\end{aligned}
$$

Putting

$$
\left(I-e^{i \theta} T^{*}\right)^{-1}=\sum_{n=0}^{\infty}\left(T^{*}\right)^{n} e^{n i \theta}
$$

and comparing the Fourier coefficients of the two terms inside the bracket, one sees that this expression vanishes identically.

THEOREM 4.3. $\tilde{\mathscr{V}}_{T} \mathscr{V}_{T}=\mathscr{V}_{T} \tilde{\mathscr{V}}_{T}$ if and only if $T$ is normal.

\section{Proof.}

$$
\begin{aligned}
& \mathscr{V}_{T}\left(e^{i \theta}\right) \mathscr{V}_{T}^{\chi}\left(e^{i \theta}\right)-\tilde{\mathscr{V}}_{T}\left(e^{i \theta}\right) \mathscr{V}_{T}\left(e^{i \theta}\right) \\
& =\left(I-T^{*} T\right)^{-1 / 2}\left(e^{i \theta}-T^{*}\right)\left(I-e^{i \theta} T\right)^{-1}\left(e^{i \theta}-T\right)\left(I-e^{i \theta} T^{*}\right)^{-1}\left(I-T^{*} T\right)^{1 / 2} \\
& \quad-\left(I-T T^{*}\right)^{1 / 2}\left(e^{i \theta}-T\right)\left(I-e^{i \theta} T^{*}\right)^{-1}\left(e^{i \theta}-T^{*}\right)\left(I-e^{i \theta} T\right)^{-1}\left(I-T T^{*}\right)^{1 / 2}
\end{aligned}
$$

Evaluating at $z=0$ we get

$$
\left(I-T^{*} T\right)^{-1 / 2}\left(T^{*} T\right)\left(I-T^{*} T\right)^{1 / 2}=\left(I-T T^{*}\right)^{1 / 2}\left(T T^{*}\right)\left(I-T T^{*}\right)^{1 / 2} .
$$

Since $T^{*} T$ and $T T^{*}$ are selfadjoint operators the three terms on the left commute with each other, as do the terms on the right. Thus we get $T^{*} T=T T^{*}$.

5. Spectral inner functions. We turn now to the problem of characterizing those inner functions which have eigenfunctions. Our best result in this direction (Theorem 5.4) is finite dimensional.

LemMA 5.1. Let $\mathscr{U}$ be a finite dimensional inner function and let $q \in \sigma(\mathscr{U})$. Then there exists $F \neq 0, F \in H_{\mathscr{H}}^{2}$, such that $\mathscr{U} F=q F$.

Proof. Let $A=\mathscr{U}-q I$. Then $A F=0$ in $H_{\mathscr{H}}^{2}$ if and only if $\left(F, A^{*} G\right)=0$ a.e. for all $G \in H_{\mathscr{H}}^{2}$, where $(\cdot, \cdot)$ is the inner product of $\mathscr{H}$. The dual of a theorem of Cambern [5, p. 93] asserts that if $B$ is any conjugate analytic finite dimensional operator valued function, then the pointwise orthogonal complement of the range of $B$ is the range of an analytic operator valued function. Letting $B=A^{*}$, and 
noting that $\operatorname{det} B=0$ implies that the pointwise complement of the range of $B$ is nontrivial, the lemma follows.

A direct proof of the above can be given by finding explicit formulas in terms of subdeterminants of $\mathscr{U}-q I$ for $f_{1}, f_{2}, \ldots, f_{n}$, where $F=f_{1} e_{1}+f_{2} e_{2}+\cdots+f_{n} e_{n}$, the $e_{j}$ 's being an orthonormal basis for $\mathscr{H}$. The author thanks the referee for pointing out the applicability of Cambern's theorem.

Definition 5.2. The multiplicity of an eigenfunction $q$ of $\mathscr{U}$ is the dimension of the smallest range function $\mathscr{J}$ such that

where

$$
\left\{F \in H_{\mathscr{H}}^{2}: \mathscr{U} F=q F\right\} \subset \mathscr{M}_{\mathscr{J}}
$$

$$
\mathscr{M}_{\mathscr{J}}=\left\{F \in L_{\mathscr{H}}^{2}: F\left(e^{i x}\right) \in \mathscr{J}\left(e^{i x}\right) \text { a.e. }\right\} \text {. }
$$

A range function $\mathscr{J}$ is a mapping from the unit circle to closed subspaces of $\mathscr{H}$. $\mathscr{J}$ is said to be analytic if there is a denumerable family $\left\{F_{j}\right\}$ of elements of $H_{\mathscr{H}}^{2}$ such that $\mathscr{J}\left(e^{i x}\right)$ is a.e. the closed span of the vectors $\left\{F_{j}\left(e^{i x}\right)\right\}$. In this case it is known that we can find an at most denumerable family $\left\{E_{j}\right\} \subset H_{\mathscr{H}}^{2}$ such that $\left\{E_{j}\left(e^{i x}\right)\right\}$ is a.e. an orthonormal basis for $\mathscr{J}\left(e^{i x}\right)$. The number of $E$ 's is then the dimension of $\mathscr{J}$. In particular, if $\mathscr{J}$ is analytic, the dimension of $\mathscr{J}\left(e^{i x}\right)$ is a.e. constant. For proofs and further information consult [5, pp. 65-66, 91-99] or [7, pp. 207, 210-212].

Definition 5.3. A finite dimensional inner function $\mathscr{U}$ is spectral if the sum of the multiplicities of its eigenfunctions is the dimension of $\mathscr{H}$.

THEOREM 5.4. A unitary operator valued function $\mathscr{U}$ is spectral if and only if $\mathscr{U}$ is inner and $\mathscr{U}=\mathscr{V} \mathscr{D} \mathscr{V}^{*}$, where $\mathscr{D}$ is a diagonal inner function and $\mathscr{V}$ is an arbitrary unitary operator valued function. If $\mathscr{U}$ is spectral $\mathscr{V}$ can be chosen to be inner with outer columns.

Proof. Sufficiency is clear. Let $q_{1}, q_{2}, \ldots, q_{n}$ be the eigenfunctions of $\mathscr{U}$ and let $\mathscr{M}_{j}=\left\{F \in H_{\mathscr{H}}^{2}: \mathscr{U} F=q_{j} F\right\}$. Then each $\mathscr{M}_{j}$ is an invariant subspace and we choose Lax vectors [5, pp. 60-63] $E_{1}, E_{2}, \ldots, E_{N}$, where the first $n_{1}$ of these belong to $\mathscr{M}_{1}$, the second $n_{2}$ to $\mathscr{M}_{2}$, and so forth. In other words $E_{1}, E_{2}, \ldots, E_{n_{1}}$ are the columns of the partial isometry $\mathscr{U}_{1}$ such that $\mathscr{U}_{1} H_{\mathscr{H}}^{2}=\mathscr{M}_{1}$. It is clear that the $E$ 's are outer; i.e., if we define a range function $\mathscr{J}_{i}$ by $\mathscr{L}_{i}\left(e^{i x}\right)=$ linear space of $E_{i}\left(e^{i x}\right)$, then $\mathscr{M}_{\mathscr{J}_{i}} \cap H_{\mathscr{H}}^{2}$ is the invariant subspace spanned by $E_{i}$. One checks easily that $\mathscr{U} \mathscr{V}=\mathscr{V} \mathscr{D}$, where $\mathscr{V}$ is the inner function whose $i$ th column is $E_{i}$.

DEFINITION 5.5. A scalar inner function $q$ belongs to the point spectrum of $\mathscr{U}$ if there exists $F \not \equiv 0, F \in H_{\mathscr{H}}^{2}$, such that $\mathscr{U} F=q F$.

The following should then be clear.

THEOREM 5.6. (i) If $\mathscr{U}$ has point spectrum $q_{1}, q_{2}, \ldots$ whose eigenvectors span $\mathscr{H}$ a.e., then $\mathscr{U}=\mathscr{V} \mathscr{D} \mathscr{V}^{*}$ where $\mathscr{D}$ is the infinite matrix $\operatorname{diag}\left(q_{1}, q_{2}, \ldots\right)$ and $\mathscr{V}$ is inner.

(ii) If $\mathscr{U}$ has eigenvectors $E_{1}, E_{2}, \ldots$ then $\mathscr{U}=\mathscr{V} \mathscr{D} \mathscr{V}^{*}$, where $\mathscr{D}$ is diagonal when restricted to the subspaces spanned by the eigenvectors. 
Finally, we note that while it is possible to construct $N$ dimensional inner functions whose eigenfunctions have total multiplicities equal to any integer between 1 and $N-2$, the following result, especially useful when $N=2$, is valid.

THEOREM 5.7. If $\mathscr{U}$ has eigenfunctions $q_{1}, q_{2}, \ldots, q_{k}$ of multiplicities $n_{1}, n_{2}, \ldots, n_{k}$ where $n_{1}+n_{2}+\cdots+n_{k}=N-1$ then

$$
q=\frac{\operatorname{det} \mathscr{U}}{\left(q_{1}\right)^{n_{1}} \cdots\left(q_{k}\right)^{n_{k}}}
$$

is an eigenfunction and $\mathscr{U}$ is spectral.

Proof. Let $\mathscr{M}_{j}=\left\{F \in H_{\mathscr{H}}^{2}: \mathscr{U}_{j} F=q_{j} F\right\}$. Then $\mathscr{M}_{1} \oplus \cdots \oplus \mathscr{M}_{k}$ is pointwise invariant under the normal operator $\mathscr{U}\left(e^{i \theta}\right)$, for almost all $\theta$. Thus for almost all $\theta$, the one dimensional perpendicular complement in $\mathscr{H}$ is invariant, and therefore an eigenvector with pointwise eigenvalue $q\left(e^{i \theta}\right)$. Thus $q$ is an eigenfunction and it follows from Theorem 5.1 that we can choose the pointwise eigenvectors so as to define an element of $H_{\mathscr{H}}^{2}$.

We conclude by mentioning some new problems. An invariant subspace determines an inner function only up to a constant inner factor on the right [5, p. 64], and since the class of all inner functions of the form $\mathscr{D} \mathscr{U} \mathscr{C} \mathscr{D}^{*}$, where $\mathscr{C}, \mathscr{D}$ are constant inner functions, is the same as the class of all inner functions of the form $\mathscr{C} \mathscr{U} \mathscr{D}$, the following concept is natural.

DefiNition 5.8. Inner functions $\mathscr{U}, \mathscr{V}$ are equivalent if there exist constant inner functions $\mathscr{C}, \mathscr{D}$ such that $\mathscr{U}=\mathscr{C} \mathscr{V} \mathscr{D}$.

The basic question is, when are $\mathscr{U}$ and $\mathscr{V}$ equivalent. None of the concepts formulated in this paper seem to help. $\mathscr{U}$ can have eigenfunctions, but not $\mathscr{V} . \mathscr{U}$ can be of scalar type, but not $\mathscr{V}$.

\section{REFERENCES}

1. A. Beurling, On two problems concerning linear transformations in Hilbert space, Acta Math. 81 (1949), 239-255.

2. N. Dunford and J. T. Schwartz, Linear operators, Part 1, Interscience, New York, 1958.

3. P. R. Halmos, Shifts on Hilbert spaces, J. Reine Angew. Math. 208 (1961), 102-112.

4. - Introduction to Hilbert Space, Chelsea, New York, 1950.

5. H. Helson, Lectures on invariant subspaces, Academic Press, New York, 1964.

6. H. Helson and D. Lowdenslager, Prediction theory and Fourier series in several variables, Acta Math. 99 (1958), 165-202.

7. —_ Prediction theory and Fourier series in several variables. II, Acta Math. 106 (1961), 175-213.

8. K. Hoffman, Banach spaces of analytic functions, Prentice-Hall, Englewood Cliffs, N. J., 1962.

9. P. D. Lax, Translation invariant subspaces, Acta Math. 101 (1959), 163-178.

10. P. D. Lax and R. S. Phillips, Scattering theory, Bull. Amer. Math. Soc. 70 (1964), 130-142.

11. S. Lefschetz, Introduction to topology, Princeton Univ. Press, Princeton, N. J., 1949. 
12. V. P. Potapov, The multiplicative structure of J-contractive matrix functions, Amer. Math. Soc. Transl. (2) 15 (1960), 131-243.

13. G.-C. Rota, Note on the invariant subspaces of linear operators, Rend. Circ. Math. Palermo (2) 8 (1959), 182-184.

14. I. E. Segal, Decompositions of operator algebras. II, Mem. Amer. Math. Soc., No. 9, 1951.

15. M. J. Sherman, Operators and inner functions, Pacific J. Math. 22 (1967), 159-171.

UNIVERSITY OF CALIFORNIA,

Los ANgeles, California 